\title{
Liquid Chromatography-Fluorescence
}

National Cancer Institute

\section{Source}

National Cancer Institute. Liquid Chromatography-Fluorescence. NCI Thesaurus. Code C122169.

An analytical technique where liquid chromatography with a fluorescence detector is used to separate, identify, and quantify substances in a sample. 